\title{
Imaging artificial membranes using high-speed Atomic Force Microscopy
}

\author{
Hussein Nasrallah ${ }^{1,2}$, Anthony Vial ${ }^{1,2}$, Nicolas Pocholle ${ }^{3}$, Jérémy Soulier ${ }^{1,2}$, Luca Costa $^{1,2}$, Cédric \\ Godefroy $^{1,2}$, Eric Bourillot ${ }^{3}$, Eric Lesniewska ${ }^{3}$, and Pierre-Emmanuel Milhiet ${ }^{1,2, \S}$
}

\author{
${ }^{1}$ INSERM, Unité 1054, 34090 Montpellier, France \\ ${ }^{2}$ Université de Montpellier, CNRS, UMR 5048, Centre de Biochimie Structurale, 34090 Montpellier, \\ France \\ ${ }^{3}$ ICB UMR CNRS 6303, University of Bourgogne Franche-Comte, Dijon, F-21000, France \\ $\S$ to whom correspondence should be addressed, pem@cbs.cnrs.fr
}

\section{Summary}

Supported lipid bilayers represent a very attractive way to mimic biological membranes, especially to investigate molecular mechanisms associated with the lateral segregation of membrane components. Observation of these model membranes with high-speed atomic force microscopy (HS-AFM) allows the capture of both topography and dynamics of membrane components, with a spatial resolution in the nanometer range and image capture time of less than 1s. In this context, we have developed new protocols adapted for HS-AFM to form supported lipid bilayers on small mica disks using the vesicle fusion or Langmuir-Blodgett methods. In this chapter we describe in detail the protocols to fabricate supported artificial bilayers as well as the main guidelines for HS-AFM imaging of such samples.

Key words: atomic force microscopy; lipid; artificial membrane, supported lipid bilayer, vesicle fusion, Langmuir, dynamics

Running title: High-Speed AFM of Membranes 


\section{Introduction}

Cell membranes are very complex structures, consisting of a multitude of different lipids and proteins. They are essential for life, delineating intracellular compartments or forming a protective barrier for the cell, such as the plasma membrane of eukaryotic cells. By organizing its components in space and in time, cell plasma membranes play a pivotal role in many biological processes including cell recognition, signalling, selective-ion transfer, adhesion and fusion. In order to investigate the molecular mechanisms associated to these functions, the complexity of biomembranes has led over the past years to the use of model membranes. By simplifying the system, it is possible to systematically study the sub-components of cellular membranes and therefore gain valuable insights into the lateral segregation of membrane components and their implications in membrane remodeling. There are currently two main categories of model membranes: i) free-standing membranes like giant unilamellar vesicles (GUV) that are very popular to study dynamic events and have been widely used to explore lipid domain formation using single molecule optical microscopy [1]. This approach, however, is restricted by the diffraction-limited resolution and is therefore not suitable to probe membrane on the mesoscopic scale; ii) planar model membranes supported by a substrate and named supported lipid bilayer (SLB)[2]. The substrates are generally glass, silicon or mica. SLBs are robust systems that are compatible with most surface-based biophysical techniques including fluorescence microscopy, ellipsometry and atomic force microscopy (AFM).

AFM is nowadays recognized as an outstanding technique in the membrane field [3]. Vertical and lateral resolution in the nanometer range can be achieved in buffer, a great advantage as compared to other structural biology techniques. In addition, due to a high signal to noise ratio, AFM often does not require image averaging, limiting the number of acquisitions required. AFM has been widely used to probe both the topography at the nanoscale of artificial SLBs [4] and their physical properties [5]. Major advances have been made in the understanding of lateral organization in biological membranes using SLBs analyzed by AFM. Lipid membrane microdomains and the importance of certain lipids such as cholesterol (Chl), gangliosides or ceramides have been characterized using AFM [6-8]. Membrane partition of proteins associated with membrane microdomains have also been measured by AFM imaging of lipid phaseseparated membranes, e.g. GPI-anchored alkaline phosphatase (reviewed in [9]). In addition to its use in characterizing membrane microdomains, AFM is suitable to acquire images of proteins reconstituted within SLB with subnanometer resolution such as the light-harvesting complex of bacterial photosynthetic 
apparatus [10] or pore-forming proteins such as cytolysin [11]. Finally AFM has been applied in membrane-bioinspired nanotechnology [12].

Conventional AFM is limited by the time to acquire one image, in the minute range, and AFM imaging was therefore unable to provide information about the dynamics of most biological processes. However tremendous progresses in AFM during the last 10 years enabled the development of high-speed (HS)-AFM able to capture images of fragile biological samples up to the video rate [13]. Early developments came from the group of Paul Hansma and Toshio Ando in the early 90s. The latter pushed the limits of AFM speed and developed a new setup that is now commercially available (see the history of HS-AFM developments in [14]). Main advances reside in the development of small cantilevers oscillating in the $\mathrm{MHz}$ range in liquid and new feedback control technique such as dynamic PID control [13]. Nowadays the majority of companies offer new microscopes allowing fast AFM imaging in the range of one frame per second. High-speed AFM has been already used to visualize in real time the formation of SLB from tubular membranes composed of a ternary phospholipid mixture with a sampling rate better than 1 image per second [15]. Real-time visualization of the assembly of a sphingomyelin-specific pore-forming toxin on planar lipid membranes was also performed, this protein forming hexagonal close-packed structures whose assembly was driven by reorganization of lysenin oligomers including association/dissociation and rapid diffusion along the membrane [16]. More recently, thanks to the development of temperaturecontrolled HS-AFM, it was possible to observe both topography and dynamics of lipid phase transition from ripple phase to fluid phase [17].

In this chapter, we detail two protocols for preparing SLBs suitable for HS-AFM as well as imaging conditions and microscope tuning. Examples of real time imaging of model membranes are also provided.

\section{Materials}

\subsection{Supplies}

1. $2 \mathrm{~mm}$ diameter glass rods from RIBM (Research Institute of Biomolecule Metrology Co., Ltd., Tsukuba, Japan)

2. Muscovite mica from Goodfellow (France) or from RIBM Co. (Japan)

3. Polyether ether ketone (PEEK) chemically-modified with hydroxyapatite (HA) by the plasma spray technique (PEEK-OPTIMA HA, Invibio, UK)

4. Araldite ${ }^{\circledR}$ glue 
5. Silicon nitride AFM tips are from Olympus (AC10FS, $k=0.1 \mathrm{~N} / \mathrm{m}$ ) or from Nanoworld (USC-F1.2, Neuchâtel, Switzerland, $k=0.15 \mathrm{~N} / \mathrm{m}$ ). Both tips are functionalized with a carbon nano fiber (CNF).

\subsection{Reagents}

1. Lipids: Lipid reagents must be of the highest purity and can be purchased from Avanti Polar Lipids (Alabaster, USA) or other suppliers. Lipid mixtures that we used include 1,2-dioleoyl-snglycero-3-phosphocholine (DOPC), 1,2-dipalmitoyl-sn-glycero-3-phosphocholine (DPPC) and cholesterol (Chl). Lipid stock solutions are stored at $-80^{\circ} \mathrm{C}$ in $\mathrm{CHCl}_{3}$ solution or in powder and kept under argon 6-12 months according to the supplier's recommendations. Working solutions are prepared at $10 \mathrm{mM}$ in $2: 1 \mathrm{CHCl}_{3} / \mathrm{MeOH}$ and stored at $-20^{\circ} \mathrm{C}$.

2. Phosphate buffered saline (PBS) at pH 7.4; and $10 \mathrm{mM}$ Tris, $150 \mathrm{mM} \mathrm{KCl,} \mathrm{pH} 7.4$

\subsection{Instrumentation}

1. Avanti mini extruder (Avanti, USA).

2. Langmuir-Blodgett balance KSV NIMA (Biolin Scientific, Sweden)

3. Homemade $10 \times 20 \mathrm{~mm}$ dipping plate in PEEK with three holes for $1 \mathrm{~mm}$ diameter glass rods for HS-AFM

4. Standard Infuse/Withdraw Pump 11 Elite dual programmable syringe Pump (Harvard Apparatus, US) with infusion tubing polyethylene (PE-10/100) and female Luer stubs to connect infusion tubing to $150 \mu \mathrm{L}$ Hamilton syringes.

5. Infuse/Withdraw tip associated with two manual probe positioners (Probe Head DDP105, Cascade Microtech Co., USA) with a resolution of $3 \mu \mathrm{m}$ for travel range over 5-25 $\mathrm{mm}$ in order to bring the fluid on each side of the HS-AFM cantilever holder.

6. HS-AFM was performed with a prototype from Toshio Ando's laboratory and provided by RIBM Research Institute of Biomolecule Metrology Co., Ltd., Tsukuba, Japan). The microscope can be equipped with of 1,5 or $40 \mu \mathrm{m}$ scanner (respectively $x=1 \mu \mathrm{m} / \mathrm{y}=4 \mu \mathrm{m} / \mathrm{z}=1 \mu \mathrm{m}, \mathrm{x}=5 \mu \mathrm{m} / \mathrm{y}=5 \mu \mathrm{m} / \mathrm{z}=2$ $\mu \mathrm{m}$ and $\mathrm{x}=40 \mu \mathrm{m} / \mathrm{y}=40 \mu \mathrm{m} / \mathrm{z}=6 \mu \mathrm{m})$.

7. RF plasma etcher (Diener, Intco France). 
8. HS-AFM cantilever holder for cantilever etching (homemade).

\section{Methods}

\subsection{Preparation of artificial supported lipid bilayers for HS-AFM}

\subsubsection{Vesicle fusion method}

Different methods have been developed to prepare SLB but the most popular remains the formation of supported membranes by fusion of large unilamellar lipid vesicles (LUVs) on a solid surface [18]. LUVs are prepared via extrusion and the vesicle solution is then added on top of the support. Vesicles then adsorb on the substrate before rupturing and form a continuous supported bilayer. We often use phaseseparated lipid bilayers, (e.g. DOPC/DPPC SLBs) in order to assess the formation of a continuous membrane (phase separation is easily observed with AFM [19]. DOPC is known to have a transition temperature of $16.5{ }^{\circ} \mathrm{C}$ and therefore exists in the fluid like liquid crystalline state $(L \alpha)$ at room temperature whereas DPPC has a transition temperature of $41.3^{\circ} \mathrm{C}$ and therefore exists in the solid-like gel state $(L \beta)$ at this temperature. The main steps of the protocol described below are for HS-AFM experiments. The protocol for conventional AFM has already been published in another Methods in Molecular Biology issue [10].

1. Glue a $2 \mathrm{~mm}$ diameter mica disk with Araldite $^{\circledR}$ at the top of a glass rod and let it dry at least overnight.

2. Add the lipids solubilized in organic solvent flow in a $\operatorname{Pyrex}^{\circledR}$ tube at $30^{\circ} \mathrm{C}$ and $\mathrm{dry}$ them under nitrogen using a dry block for at least 2 hours. If a lipid mixture is used, vortex the tube before drying.

3. Suspend the dried lipids in $0.125 \mathrm{mM}$ PBS buffer by 1 min strong vortexing under argon in the presence of $2 \mathrm{~mm}$ glass beads to form multilamellar vesicles (MLV). Alternatively, longer vortexing without beads can be used.

4. Extrude MLVs using a suitable extruder (e.g., Avanti Mini-Extruder) through a $100 \mathrm{~nm}$ diameter polycarbonate membrane to produce LUVs. Their homogeneity can be easily controlled using dynamic light scattering. 
5. There are two possible methods to deposit the SLB from the LUV suspension. Method A: Cleave the small mica disk glued at the top of the glass rod under a binocular to assess the quality of cleavage, deposit $2 \mu \mathrm{L}$ LUV suspension onto it (see Fig. 1A) and allow the LUVs to adsorb and fuse during $35 \mathrm{~min}$ at $60^{\circ} \mathrm{C}$ in the oven for a DOPC/DPPC mixture (perform the incubation at $20^{\circ} \mathrm{C}$ above the fusion temperature of the lipids allowing them to form a homogeneous fluid phase). The sample is then cooled down for $5 \mathrm{~min}$ at room temperature and then rinsed with PBS. This procedure however led to some problems of reproducibility in the quality of the membrane (shape of DPPC domains, holes, double membrane) that we interpret as the consequence of the partial evaporation of the tiny drop upon incubation at $60^{\circ} \mathrm{C}$. This evaporation is difficult to control and can change salt and lipid concentration influencing the fusion process (Note 1 below). The protocol described in Fig. 1B prevents such drawbacks. Method B: A large mica disc of about $1 \mathrm{~cm}$ is punched in the centre leaving a hole of $2 \mathrm{~mm}$ diameter that will hold the glass rod with the small mica disc glued on top. After being cleaved, holding the large disc by tweezers so that the glass rod is not affected or contaminated, both will be inserted sidewise into the bottom of a $2 \mathrm{ml}$ microtube and covered by $50 \mu \mathrm{L}$ of lipids. This tube was then incubated (standing vertically) under the conditions described above $\left(60^{\circ} \mathrm{C}\right.$, in a water bath). The tube is then cooled down for $5 \mathrm{~min}$ at room temperature and then the sample rinsed with PBS.

6. After rinsing the glass rod is glued to the top of the $z$ piezo of the scanner (schematized in Fig. 1B) using nail polish or Loctite ${ }^{\circledR}$.

7. The piezo scanner is then placed over the liquid cell holding the cantilever chip according the detailed protocol provided by Uchihashi et al [20].

\subsubsection{Langmuir balance}

The main drawback of the vesicle fusion method is the symmetry of SLBs that are obtained, imperfectly mimicking biological membranes. Another approach to form SLBs on a solid support is the use of the Langmuir-Blodgett or Langmuir Schaefer techniques. Both consist of the transfer of a lipid monolayer (inner leaflet) onto a hydrophilic support by pulling it vertically through a lipid monolayer at the air-water interface. The outer leaflet is then transferred using either another vertical passage of the support through 
the lipid monolayer at the air-water interface (Blodgett) or by dipping horizontally the support into the lipid monolayer at the air-water interface (Schaefer). The advantage of the double transfer methods is that asymmetrical bilayers can be produced. We describe below a protocol to produce SLBs supported on mica which are suitable for HS-AFM imaging (Figure 3):

1. Install the Langmuir-Blodgett apparatus on a vibration isolation table and inside a complete enclosure box with temperature control $\left(22^{\circ} \mathrm{C}\right.$ in our case).

2. Before an experiment and after each run, it is crucial to clean the Langmuir trough and barriers. Wash with hot water and a commercial detergent and always use gloves to manipulate each element in order to minimise contamination.

3. Use a soft brush soaked with ethanol for mechanical and chemical cleaning of LB elements. Rinse thoroughly with Milli-Q water (18M $\Omega)$.

4. Clean the platinum Wilhelmy plate over a blue flame (Bunsen burner) hot enough to make the plate glow red in just a few seconds. Rinse with ethanol and water.

5. Place the trough, the barriers and the Wilhelmy plate in the frame.

6. Solvent quality and its nature (volatile or not) impact the Langmuir isotherm. Equilibrium time and subphase temperature should be maintained constant during the process.

7. Fill the LB trough with pure water (the subphase) in order to to get a positive meniscus of $2 \mathrm{~mm}$ high on the Wilhelmy plate.

8. Open the barriers and zero the LB balance (associate to the Wilhelmy plate).

9. Close the barriers. The surface pressure should not change (less than $0.2 \mathrm{mN} / \mathrm{m}$ ). If the surface pressure change, it is caused by contaminants. A new cleaning process should be performed.

10. We designed and used a specific hydrophilic and bio-inert holder (PEEK treated with hydroxyapatite) to dip three HS-AFM mica substrates at the same time (Figure 3B). It was also cleaned in the same way as the LB barriers. Cleave the three mica disks and immerse the dipping system just before adding the lipids (see below).

11. Fill a precision pipette-puller with a suitable volume ( $20 \mu \mathrm{L}$ of lipids in our case) of the prepared solution of lipids in a volatile organic solvent (chloroform). The organic solvent should be non-polar to prevent miscibility in water and volatile so that it will evaporate at the air-water interface. 
12. Gently add the lipids at the interface by depositing the drop in contact with water (do not let the drop fall down from the needle as part of the sample might be lost in the subphase as micelles). Do not touch the water with the needle.

13. Wait $15-20 \mathrm{~min}$ for the pressure surface to reach equilibrium (the time for the solvent to evaporate).

14. Start the experiment according to the protocol provided by the LB equipment manufacturer (see an example in [21]:

- Compress the lipid film at a rate of $5 \mathrm{~cm}^{2} / \mathrm{min}$ up to a pressure mimicking that of a biological membrane (typically $30-35 \mathrm{mN} / \mathrm{m}$ ). The maximum pressure for the $L B$ film collapsing should determined when using a new lipid mixture.

- The dipping mechanism holds the substrate and enables controlled deposition cycles. Monolayers should be deposited at a constant surface pressure of 30 $\mathrm{mN} / \mathrm{m}$, after a 5-min relaxation time, by raising vertically $(0.5 \mathrm{~mm} / \mathrm{min})$ freshly cleaved mica through the air-water interface (Figure $3 \mathrm{C}$ ).

- The deposited sample is left $30 \mathrm{~min}$ in a desiccator under nitrogen and in darkness in order to limit oxidation of unsaturated aliphatic chains and a second deposition is performed in order to get a second leaflet producing a bilayer.

\subsection{HS-AFM Imaging}

The procedure described in 3.1.1 led to a flat supported lipid bilayer with a root mean square ( $\mathrm{rms}$ ) in the Angstrom range (see examples in Fig. 2). In order to image such SLB at scanning rate up to 10 images/s, we used the HS-AFM setup developed by Toshio Ando's group in oscillating mode with optimized highresolution imaging parameters to minimize the force applied by the tip on the sample, an important parameter for biological AFM imaging. Typically, short cantilevers designed for HS-AFM (length < $10 \mu \mathrm{m}$, a spring constant of $0.1-0.2 \mathrm{~N} / \mathrm{m}$, a resonance frequency of $600 \mathrm{kHz}$ and a quality factor of $\sim 2$ in solution) were oscillated at free amplitude of a few $\mathrm{nm}$ (below $0.5 \mathrm{~V}$ amplitude voltage). The amplitude set point was set at $80-90 \%$ of the free amplitude. The scanning rate was generally 1 or 2 frames/s with a $200 \times 200$ pixels sampling but largely depended on the scanner used (basically, we were not able to achieve more than 1 image/s with the $40 \mu \mathrm{m}$ scanner) and on the scanning area (generally 4-5 $\mu \mathrm{m}$ for observing SLB structure). Another important feature is the presence of an electron beam deposited tip at the end of the 
cantilever [20]. It is very important for high-resolution imaging since this EBD tip can be sharpened using plasma etching, and it also increases the distance between the sample and the cantilever, minimizing hysteresis during fast scanning.

Using this setting, we have been able to image in real time the formation of SLB composed of an equal mixture of DOPC and DPPC. We observed the formation of the SLB from unilamellar vesicles that can be identified in the HS-AFM images (Figure 1, A to D). Over time we observed the fusion of vesicles on mica leading to formation of a flat supported membrane. Vesicle fusion was however too fast for HS-AFM to capture intermediate shapes of vesicle during this process. Importantly we cannot completely exclude that part of this fusion is induced or favored by tip scanning. This hypothesis is in good agreement with the pattern observed in Fig. $2 \mathrm{C}$ where the vesicles seems to be partially fused with a separation between the 2 states along the scan axis. The picture in Fig. 2E shows a classical pattern of DOPC-DPPC membrane with three domains: a light domain corresponding to DPPC enriched domains in gel phase protruded from a darker matrix made of DOPC enriched fluid domains (white arrow). The height difference between the two domains was $1.2 \pm 0.2 \mathrm{~nm}$ and fitted well with lipid phase separation between fluid and gel phases. The darkest domain corresponded to the mica substrate that is around $4-5 \mathrm{~nm}$ lower than the DOPC domain, a value that fits well with the thickness of a lipid bilayer with a thin layer of buffer between the lipid polar heads and the substrate. Importantly this pattern cannot be obtained just after the vesicle fusion since phase separation requires lipids to laterally segregate according to their physical properties [22]. During this membrane remodelling, HS-AFM imaging can be difficult due to lipid diffusion.

It is well documented that components of biological membranes are very dynamic and HS-AFM represents a very attractive tool to monitor $2 \mathrm{D}$-diffusion in real time. It has already been used to investigate diffusion and dynamic interaction of membrane proteins [13] and a recent paper described the dynamics of lipid phases during transition [17]. HS-AFM is therefore an outstanding tool to probe cholesterol effects in membrane organization. Indeed this lipid plays a major role in the regulation of the fluidity and mechanical stiffness of membranes [23]. This ability of cholesterol to modulate the fluidity of membranes is thought to be important in the formation of membrane microdomains [24] and associated to many biological processes, including cell fusion [25] or development of Alzheimer's disease [26]-[27] (in fact, the biological significance of cholesterol-induced changes in membrane fluidity is vast, and these reflect only a small proportion of affected areas). A glance into the benefit of using HS-AFM for imaging Chlcontaining SLB is shown in Figure 2 (right column) describing Chl effect on the basic DOPC/DPPC mixture. 
Using the vesicle fusion method, a mixture composed of DOPC/DPPC/Chl (1:1:0.35) led to the formation of ordered lipid domains surrounded by a fluid phase. The shape of ordered domains was a little bit different as compared to the DOPC/DPPC reference mixture with more round-shaped domains, most likely composed of DPPC in interaction with Chl. These 2 lipids are known to preferentially interact with each other as compared to the DOPC/Chl pair [28]. Chl is known to increase the fluidity of ordered domains (here DPPC) and probably reduced the line tension within the membrane, which supports the shape of ordered domains. The images captured with HS-AFM also showed that the membrane is dynamic and that DPPC/Chl domains could diffuse in the fluid DOPC matrix and even coalesce to form larger domains (black arrow in Fig. 2G).

Using the Langmuir-Blodgett technique, phase separated lipid membranes can also be observed on mica with a pattern that is similar to that obtained with SLB made using the vesicle fusion method (compare Figure $2 \mathrm{E}$ and Figure $4 \mathrm{~A}$ ). A DOPC matrix surrounded gel phase domains. SLBs obtained at $32 \mathrm{mN} / \mathrm{m}$ surface pressure covered about $80 \%$ of the whole investigated area, as shown in figures $4 A$ and $4 B$. DPPC domains were relatively stable in shape and position as compared to mica area devoid of lipids. Membrane dynamics could nevertheless be observed in some places (black asterisks in Fig. 4A and Fig. 4B). Importantly we observed that membrane mica coverage depends on surface pressure transfer. Indeed decreasing surface pressure applied to the lipid film during the transfer to $12 \mathrm{mN} / \mathrm{m}$ led to a huge decrease in membrane coverage, below $30 \%$. Under these conditions, membrane dynamics can be observed over time, e.g. DPPC domain remodeling in figures 4C and 4D. Such small membranes were very sensitive to tip scanning and membrane patches could be easily removed by the tip.

\subsection{Conclusion and perspectives}

HS-AFM is assuredly an outstanding technique to probe both structure and dynamics of membrane assemblies. The shape of lipid domains can be easy delineated with the tip scanning at several images per second and HS-AFM is also very useful to study membrane-protein interactions in real time [29]. In the future, further improvements in scanning speed would help in better understanding membrane organization since lipid diffusion is very high (in the $\mu \mathrm{m}^{2} / \mathrm{s}$ range). In addition, SLB imaging with HS-AFM would benefit from its combination with fluorescence or Raman microscopy in order to identify membrane components delineated by the tip. 


\section{Notes}

1. It is important to stress again that the small size of the sample holder is critical for SLB imaging using AFM. Some tricks similar to what we described in Fig. 1B need to be developed to prevent any evaporation or dewetting problems.

2. In Langmuir-Blodgett experiments, the optimal surface pressure obtained by plotting surface pressure-area isotherm depends on the composition in lipids of the monolayer and is established empirically (typically 30-35 mN/m for DOPC-DPPC). The surface pressure-area isotherm can also provide a measure of the average area per molecule and the compressibility of the monolayer.

3. Surface hydrophillicity of the PEEK plate can be increased by treating the dipping part by 172-nm Xenon excimer radiation to generate hydroxyl and carbonyl groups.

4. An important parameter to control is the power of the laser diode reflected on the cantilever. Therefore for laser over $0.2 \mathrm{~mW}$ for the Olympus cantilever and $0.5 \mathrm{~mW}$ for the Nanoworld cantilever, we could observe the melting of the DPPC phase, strongly suggesting that the temperature locally increases beneath the tip during imaging (no increase of temperature was recorded in the buffer bathing the cantilever). DPPC melting temperature is about $40^{\circ} \mathrm{C}$.

5. The composition of the buffer used for vesicle fusion has to be finely tuned for optimal mica coverage. For instance, adsorption of negatively charged vesicles made from a mixture of palmitoyl-oleoyl-phosphoglycerol (POPG)/palmitoyl-oleoyl-phosphatidyletha-nolamine (POPE) lipids on a mica (also negatively charged) is only possible in the presence of divalent cations [30].

6. It is sometimes useful to tune lipid concentration and fusion buffer composition for optimizing substrate coverage. The duration of SLB cooling-down can also influence vesicle fusion.

7. Tip contamination is one of the main problems, especially in HS-AFM where tip scanning is very rapid. Fortunately the EBD-functionalized tips can be easily cleaned using plasma etching [20].

8. Mica needs to be cleaved just before the addition of lipids.

\section{Acknowledgments}

The research has been supported by CNRS (PEM and EL), INSERM (PEM), Institut Carnot (EL) and by the ANR program (ANR-11-nano-009-04“, ANR-08-NANO-010-03, ANR- 08-PCVI-0003-02, the EpiGenMed 
Labex ANR-10-LABX-12-01 and the French Infrastructure for Integrated Structural Biology (FRISBI) ANR10-INBS-05). We are grateful to our collaborators involved in the project, P. Dosset, J. Kokavecz, C. Le Grimellec.

\section{References}

1. Kahya N. Targeting membrane proteins to liquid-ordered phases: molecular self-organization explored by fluorescence correlation spectroscopy. Chem. Phys. Lipids. 2006;141:158-68.

2. Sackmann E. Supported membranes: scientific and practical applications. Science. 1996;271:43-8.

3. Muller DJ. AFM: a nanotool in membrane biology. Biochemistry (Mosc.). 2008;47:7986-98.

4. El Kirat K, Morandat S, Dufrêne Y. Nanoscale analysis of supported lipid bilayers using atomic force microscopy. Biochim. Biophys. Acta. 2009;

5. Garcia-Manyes S, Sanz F. Nanomechanics of lipid bilayers by force spectroscopy with AFM: a perspective. Biochim. Biophys. Acta. 2010;1798:741-9.

6. Goksu EI, Vanegas JM, Blanchette CD, Lin WC, Longo ML. AFM for structure and dynamics of biomembranes. Biochim Biophys Acta. 2009;1788:254-66.

7. Johnston I, Johnston L. Ceramide promotes restructuring of model raft membranes. Langmuir. 2006;22:11284-9.

8. Seantier B, Giocondi M, Le Grimellec C, Milhiet P. Probing supporting model and native membranes using afm. Curr. Opin. Colloid Interface Sci. 2008;13:326-37.

9. Giocondi $M-C$, Seantier $B$, Dosset $P$, Milhiet $P-E$, Le Grimellec $C$. Characterizing the interactions between GPI-anchored alkaline phosphatases and membrane domains by AFM. Pflüg. Arch. Eur. J. Physiol. 2008;456:179-188.

10. Levy D, Milhiet P-E. Imaging of transmembrane proteins directly incorporated within supported lipid bilayers using atomic force microscopy. Methods Mol. Biol. Clifton NJ. 2013;950:343-57.

11. Czajkowsky DM, Hotze EM, Shao Z, Tweten RK. Vertical collapse of a cytolysin prepore moves its transmembrane $\beta$-hairpins to the membrane. EMBO J. 2004;23:3206-3215.

12. Yu C, Groves JT. Engineering supported membranes for cell biology. Med. Biol. Eng. Comput. 2010;48:955-63.

13. Ando T, Uchihashi T, Scheuring S. Filming biomolecular processes by high-speed atomic force 
microscopy. Chem. Rev. 2014;114:3120-88.

14. Ando T, Uchihashi T, Kodera N, Yamamoto D, Miyagi A, Taniguchi M, et al. High-speed AFM and nano-visualization of biomolecular processes. Pflugers Arch. 2008;456:211-25.

15. Giocondi MC, Yamamoto D, Lesniewska E, Milhiet PE, Ando T, Le Grimellec C. Surface topography of membrane domains. Biochim Biophys Acta. 2010;1798:703-18.

16. Yilmaz N, Kobayashi T. Visualization of Lipid Membrane Reorganization Induced by a Pore-Forming Toxin Using High-Speed Atomic Force Microscopy. ACS Nano. 2015;9:7960-7.

17. Takahashi H, Miyagi A, Redondo-Morata L, Scheuring S. Temperature-Controlled High-Speed AFM: Real-Time Observation of Ripple Phase Transitions. Small Weinh. Bergstr. Ger. 2016;12:6106-13.

18. McConnell HM, Watts TH, Weis RM, Brian AA. Supported planar membranes in studies of cell-cell recognition in the immune system. Biochim Biophys Acta. 1986;864:95-106.

19. Almeida PF, Vaz WL, Thompson TE. Lateral diffusion and percolation in two-phase, two-component lipid bilayers. Topology of the solid-phase domains in-plane and across the lipid bilayer. Biochemistry (Mosc.). 1992;31:7198-210.

20. Uchihashi T, Kodera N, Ando T. Guide to video recording of structure dynamics and dynamic processes of proteins by high-speed atomic force microscopy. Nat. Protoc. 2012;7:1193-206.

21. Milhiet PE, Domec C, Giocondi MC, Van Mau N, Heitz F, Le Grimellec C. Domain formation in models of the renal brush border membrane outer leaflet. Biophys. J. 2001;81:547-555.

22. Giocondi $M C$, Vié $V$, Lesniewska $E$, Milhiet PE, Zinke-Allmang $M$, Le Grimellec $C$. Phase topology and growth of single domains in lipid bilayers. Langmuir. 2001;17:1653-9.

23. Needham D, McIntosh TJ, Evans E. Thermomechanical and transition properties of dimyristoylphosphatidylcholine/cholesterol bilayers. Biochemistry (Mosc.). 1988;27:4668-73.

24. Brown DA, London E. Functions of lipid rafts in biological membranes. Annu. Rev. Cell Dev. Biol. 1998;14:111-36.

25. Nakanishi M, Hirayama E, Kim J. Characterisation of myogenic cell membrane: II. Dynamic changes in membrane lipids during the differentiation of mouse C2 myoblast cells. Cell Biol. Int. 2001;25:971-9.

26. Yip CM, Elton EA, Darabie AA, Morrison MR, McLaurin J. Cholesterol, a modulator of membraneassociated Abeta-fibrillogenesis and neurotoxicity. J. Mol. Biol. 2001;311:723-34.

27. Chochina SV, Avdulov NA, Igbavboa U, Cleary JP, O'Hare EO, Wood WG. Amyloid beta-peptide1-40 increases neuronal membrane fluidity: role of cholesterol and brain region. J. Lipid Res. 2001;42:1292-7.

28. Fritzsching KJ, Kim J, Holland GP. Probing lipid-cholesterol interactions in DOPC/eSM/Chol and 
DOPC/DPPC/Chol model lipid rafts with DSC and (13)C solid-state NMR. Biochim. Biophys. Acta. 2013;1828:1889-98.

29. Rangl M, Rima L, Klement J, Miyagi A, Keller S, Scheuring S. Real-time Visualization of Phospholipid Degradation by Outer Membrane Phospholipase A using High-Speed Atomic Force Microscopy. J. Mol. Biol. 2017;429:977-86.

30. Picas L, Carretero-Genevrier A, Montero MT, Vazquez-Ibar JL, Seantier B, Milhiet PE, et al. Preferential insertion of lactose permease in phospholipid domains: AFM observations. Biochim Biophys Acta. 2010;1798:1014-9.

Figure captions

Figure 1 - Formation of supported lipid bilayers from lipid vesicles. The first method $(A)$ is adapted from [20] and the second method (B) has been developed to improve the reproducibility of the fabrication. A) A mica disk is glued at the top of a glass rod and placed on a polymer pad in a Petri dish. The lipid vesicle solution is deposited on mica, incubated in an oven in a water-saturated atmosphere thanks to wet Kimwipes ${ }^{\circledR}$ in the dish closed by a lid. After rinsing, the glass rod is glued at the top of the piezo scanner shown in B.

B) A mica disk of $\sim 1 \mathrm{~cm}$ diameter with a punched hole of $2 \mathrm{~mm}$ in the center is positioned at the bottom of a $2 \mathrm{ml}$ microtube. A glass rod of $2 \mathrm{~mm}$ diameter, the HS-AFM sample holder, with a $2 \mathrm{~mm}$ mica disk glued at the top, is placed in the hole. The 2 mica disks should be at the same level. A solution of lipid vesicles is then deposited at the top of the sample and incubated in a water bath (see Materials and Methods section). After incubation, the glass rod is picked up with tweezers and glued with Loctite ${ }^{\circledR}$ glue on the $z$ piezo of the scanner.

\section{Figure 2 - Imaging supported lipid bilayer and its formation using HS-AFM.}

A to D) 2 s time lapse showing real time formation of DOPC-DPPC (1:1) SLB on mica. The black arrowhead indicates a non-fused vesicle that is surrounded by a brown matrix corresponding to an already formed SLB. From $A$ to $D$, the number of intact vesicles decreases over time and a lipid phase transition can be observed in D. The white asterisk points a defect in the membrane where no membrane is present. E) Typical pattern obtained at the end of the vesicle fusion for a DOPC-DPPC bilayer. Three regions are clearly distinguishable, mica (white asterisk), DOPC and DPPC, from the darkest to the brightest, respectively. F 
to $H)$ 2s time lapse showing a DOPC-DPPC-Chl (1:1:0.35) SLB. The dynamics of the liquid-ordered (lighter) domains in terms of shape and position and their coalescence can be observed in real time (black arrow in Fig. 1G). Scale bars are $100 \mathrm{~nm}(A$ to $D), 500 \mathrm{~nm}(E)$ and $1 \mu \mathrm{m}$ ( $F$ to $G$ ). The z scale is automatically adjusted and in the $20 \mathrm{~nm}$ range.

\section{Figure 3 - Fabrication of supported lipid bilayers using Langmuir Blodgett technique}

A) Schematic view of the Langmuir balance; B) zoom on the dipping system made in polyether ether ketone (PEEK), chemically-modified and biologically inert. Glass rods with mica disk glued at the top, similar to those shown in Figure 1, are inserted in holes in the polymer plate; C) zoom on mica, glued at the top of a glass rod and covered by a lipid monolayer.

\section{Figure 4 - HS-AFM imaging of DOPC/DPPC (1:1) SLB using Langmuir Blodgett technique}

A and B) Topography of a DOPC/DPPC bilayer supported on mica, transferred at $32 \mathrm{mN} / \mathrm{m}$ surface pressure. Three regions are clearly distinguishable, mica, DOPC and DPPC, from the darkest to the brightest, respectively. The pattern is similar to SLB obtained by vesicle fusion and membrane covered $\sim 80 \%$ mica surface. The black asterisk indicates an area where DPPC remodeling can be observed. The time between A and B is $30 \mathrm{~s}$; C to D) Topography of a DOPC/DPPC bilayer supported on mica, made at 12 $\mathrm{mN} / \mathrm{m}$ surface pressure. Membrane coverage is lower as compared to $A$ and $B$ and large mica area are devoid of membrane (dark area). The time between C and D is $30 \mathrm{~s}$. HS-AFM scan rate, 4s/frame; scale bars, $1 \mu \mathrm{m}$. 


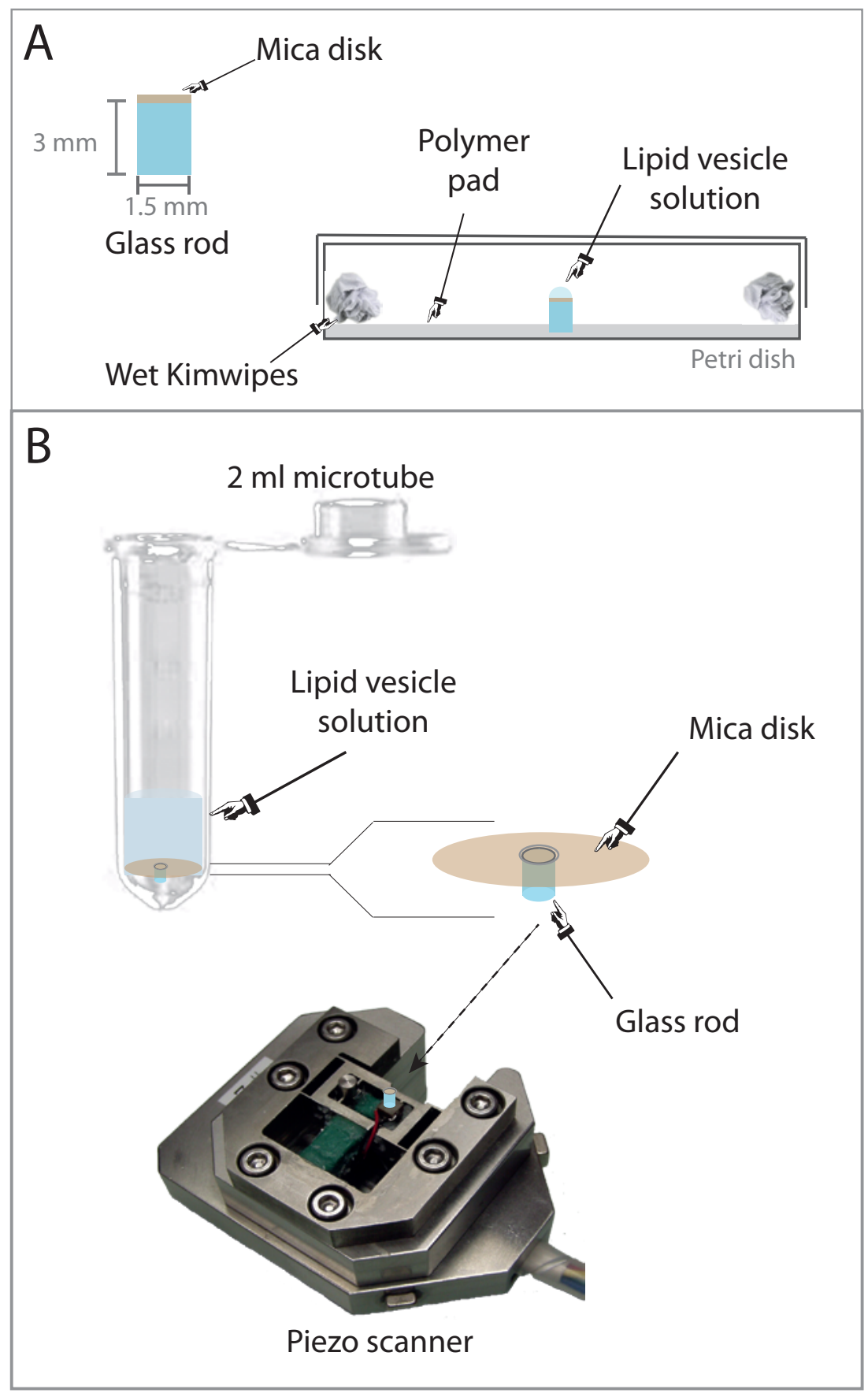




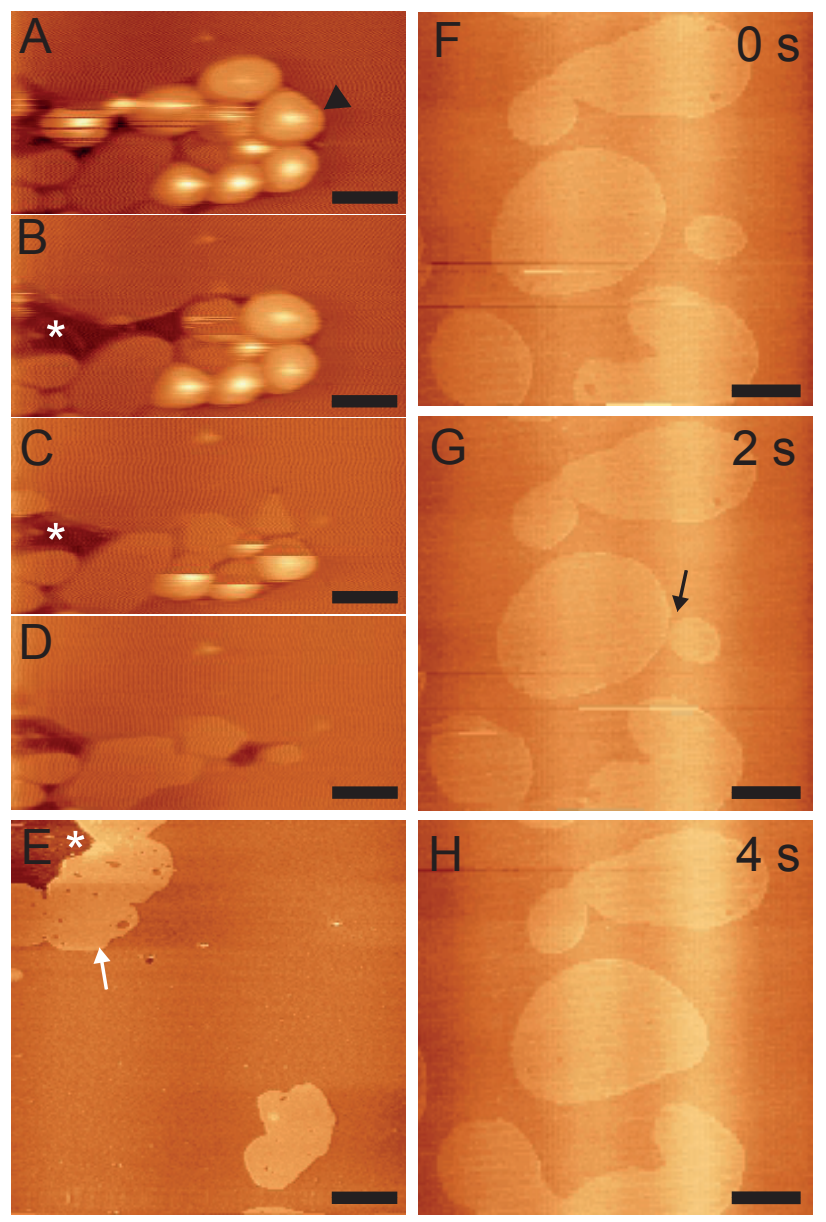




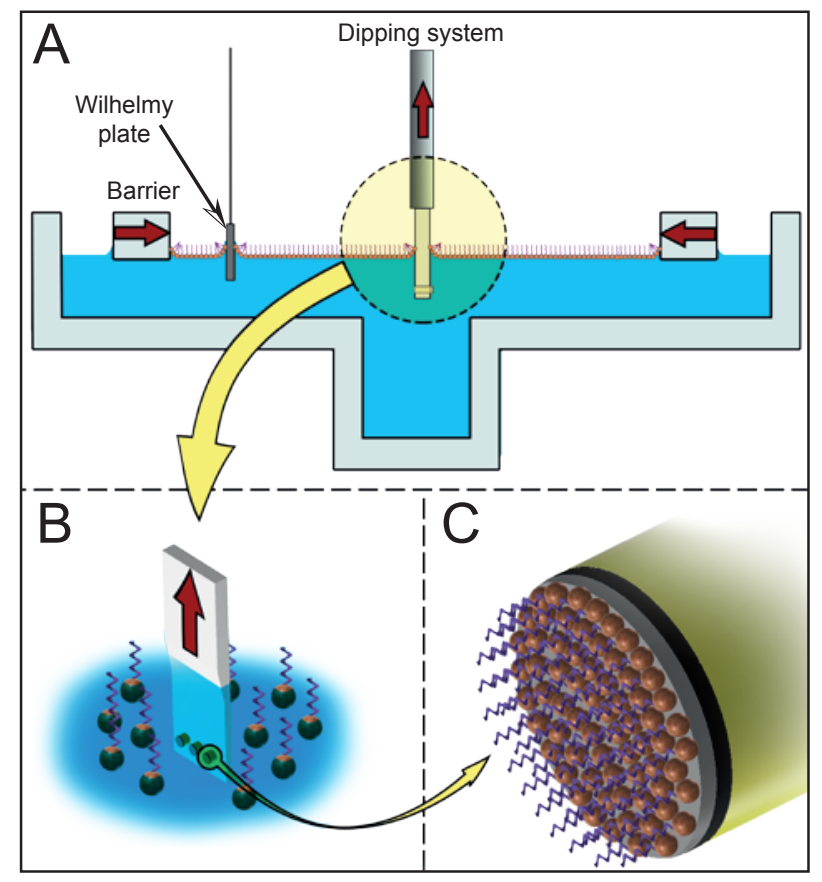



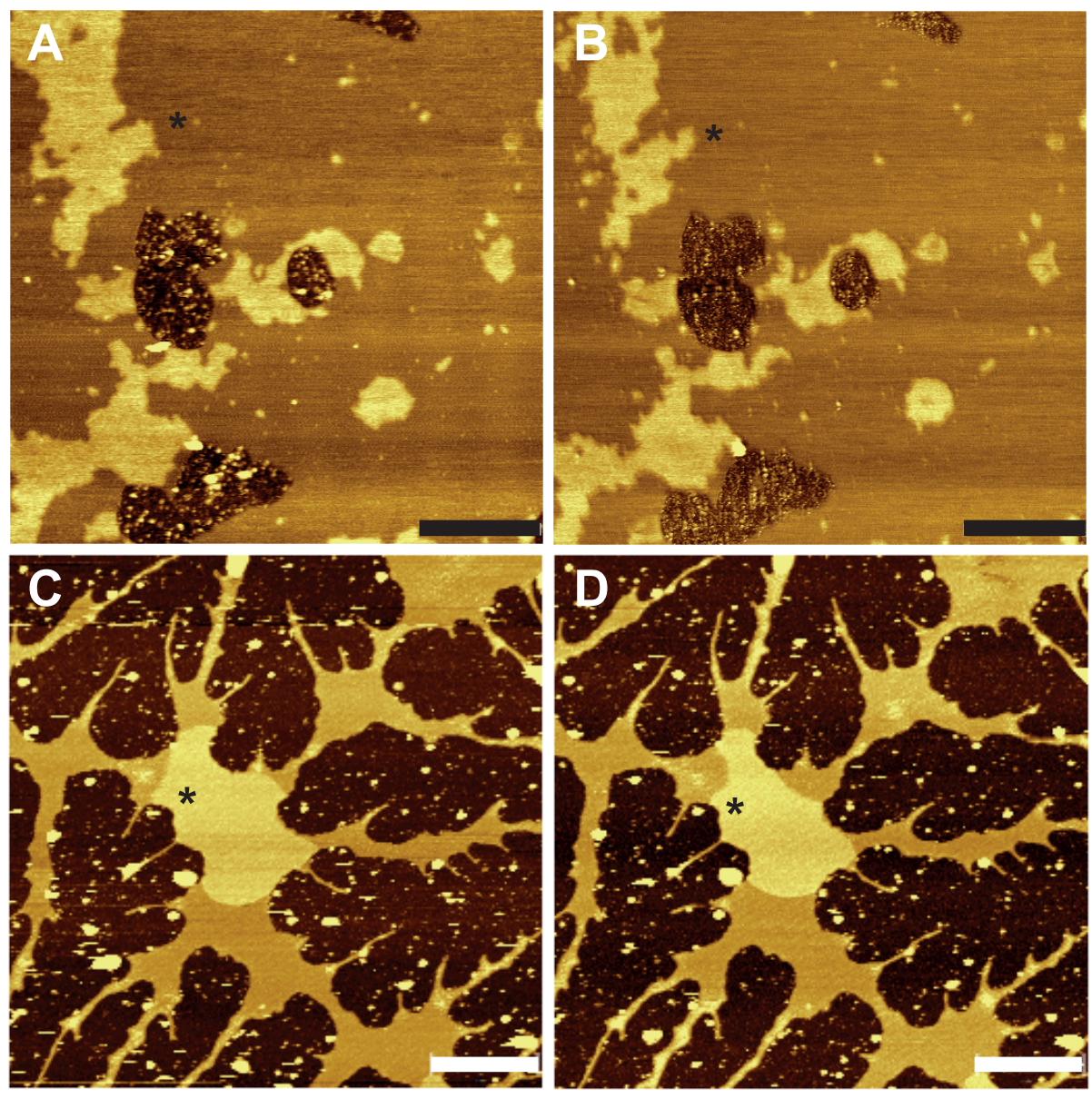